\title{
Novel mutations of the HRAS gene and absence of hotspot mutations of the BRAF genes in oral squamous cell carcinoma in a Greek population
}

\author{
DIMITRA KOUMAKI ${ }^{1}$, GEORGE KOSTAKIS ${ }^{2}$, VASILIKI KOUMAKI ${ }^{3}$, \\ NIKOLAOS PAPADOGEORGAKIS ${ }^{2}$, MICHAEL MAKRIS ${ }^{4}$, ALEXANDROS KATOULIS ${ }^{1}$, \\ SMARAGDA KAMAKARI ${ }^{5}$, GEORGE KOUTSODONTIS ${ }^{5}$, CHRISTOS PERISANIDIS $^{6}$, \\ VAIA LAMBADIARI ${ }^{7}$, EVANTHIA CHRYSOMALI $^{8}$, NIKOLAOS STAVRIANEAS $^{1}$, \\ CONSTANTINOS ALEXANDRIDIS ${ }^{2}$ and DIMITRIOS RIGOPOULOS ${ }^{1}$
}

\begin{abstract}
${ }^{1}$ Second Department of Dermatology and Venereology, Medical School, Attikon University General Hospital, Athens; ${ }^{2}$ Department of Oral and Maxillofacial Surgery, University Clinic, Evangelismos General Hospital, Dental School, University of Athens, Athens; ${ }^{3}$ Department of Microbiology, Medical School, University of Athens, Athens; ${ }^{4}$ Allergy Unit, Second Department of Dermatology and Venereology Medical School, Attikon University General Hospital, Athens; ${ }^{5}$ Biogenomica S.A., Centre of Genetic Analysis and Research, Athens; ${ }^{6}$ Department of Oral and Maxillofacial Surgery, Medical University of Vienna, Austria; ${ }^{7}$ Second Department of Internal Medicine, Research Institute Diabetes Center, Athens University Medical School, Attikon University General Hospital, Athens; ${ }^{8}$ Section of Oral Pathology and Oral Surgery, Department of Oral Pathology, Dental School, University of Athens, Athens, Greece
\end{abstract}

Received December 1, 2011; Accepted January 17, 2012

DOI: $10.3892 / o r .2012 .1653$

\begin{abstract}
Oral squamous cell carcinoma (OSCC) is the sixth most common cancer in the world. The phosphatidylinositol 3 kinase (PI3K) signalling pathway has been reported to play an important role in OSCC. Since we have previously detected absence of hotspot PIK3CA gene mutations in the Greek population, we hypothesized that BRAF or HRAS may be activated as upstream effectors of the pathway. Furthermore, the status of the HRAS and BRAF mutations in OSCC has never been assessed before in the Greek population. Eighty-six primary paraffin-embedded tumors were screened for BRAF and HRAS hotspot mutations. In HRAS, two hotspot mutations in codon $12(2.3 \%)$ and eight new genetic alterations were detected ( $8.6 \%$ overall). One new missense mutation, Alanine53Valine (Ala53Val), one silent mutation, two mutations in the 5'UTR region and four mutations in intron 1 were detected. No hotspot mutations in BRAF were found. A new silent mutation/polymorphism T1803C was detected at a percentage of $30 \%$. This study is the first to report HRAS mutations in the Greek population. The results suggest that
\end{abstract}

Correspondence to: Dr Dimitra Koumaki, Second Department of Dermatology and Venereology, Medical School, Attikon University General Hospital, Rimini 1, Athens 12462, Greece

E-mail: dkoumaki@yahoo.gr

Key words: oral squamous cell carcinoma, BRAF, HRAS
RAS is an important member of the PI3K signalling pathway and may play a role in the tumorigenesis of OSCC.

\section{Introduction}

Oral squamous cell carcinoma (OSCC) is a subset of head and neck squamous carcinoma (HNSCC) involving the oral tongue, upper gingival, lower gingival and alveolus, floor of the mouth, buccal mucosa, retromolar triangle, lip mucosa and hard palate. OSCC is considered to be the sixth most common cancer in the world (1). Smoking and alcohol consumption are major risks for HNSCC but only a fraction of people with these habits actually develop oral cancer, which implies that other genetic factors contribute to the pathogenesis of the disease $(2,3)$. Infection by human papilloma virus has also emerged as a risk factor for HNSCC (4).

The RAS family genes consist of three members, HRAS, KRAS and NRAS located on chromosomes 11, 12 and 1, respectively, encoding a $21 \mathrm{kDa}$ membrane protein (p21), which possesses GTPase activity $(5,6)$. HRAS has been shown to activate phosphatidylinositol 3 kinase (PI3K) more efficiently than KRAS and NRAS. Hotspots for RAS mutations are found in codons 12,13 and 61, causing the mutant protein to lose its ability to exchange GTP with GDP thus remaining activated (7). RAS is also is an upstream regulator of PI3K. Thus an activated RAS could also activate the PI3K signalling pathway, which is shown to be activated in oral cancer $(8,9)$. The incidence of RAS mutations varies greatly among different human tumors and different ethnicities. HRAS mutations are primarily found in cancers of the urinary tract and bladder. The incidence of RAS mutations in OSCC shows 
racial disparity, being more frequent in India and South East Asia and less common in the West (10-12).

$\mathrm{BRAF}$ is one of the most commonly mutated oncogenes in human cancer. BRAF is a serine/threonine kinase of the RAF family, which consists of three genes, namely ARAF, BRAF and CRAF (5). More than $90 \%$ of the reported BRAF mutations occur in its kinase domain regarding a single nucleotide substitution V600E (also referred to as V599E according to the numbering system used) (13). These mutations lead to constitutive activation of BRAF, regardless of activation by RAS, which in turn leads to constitutive activation of MEK and ERK, leading to proliferation and survival of cancer cells. Furthermore, it has been widely documented that mutations affecting RAS and BRAF both in human cancers and in chemically induced mouse liver tumors seem to be mutually exclusive, suggesting the operation of alternative pathways for $\operatorname{BRAF}(14,15)$.

In view of the paucity of extensive studies regarding the status of BRAF and HRAS mutations in OSCC and to i) further assess the individual effect of the HRAS and BRAF mutations in OSCC, ii) to test whether their mutations are mutually exclusive, as in other cancers, and iii) to examine whether RAS is the activating factor of the PI3K signalling pathway previously reported $(3,8,9)$ we performed a systematic molecular study in 86 clinically and histologically well-defined malignant tumors of oral squamous cell carcinoma, previously reported to lack PIK3CA hotspot mutations (15) and correlated the mutation status of HRAS and BRAF with age, gender, stage, histological grade and prognosis.

\section{Materials and methods}

Study population. Specimens from 86 consecutive patients, who underwent a major operation as part of their cancer treatment, were collected and retrieved from the Department of Oral and Maxillofacial Surgery at the Evangelismos Hospital, University of Athens, Athens, Greece, from January 2003 to December 2007. Most of them had advanced stages of the disease. In addition, tissue samples of the oral cavity from 80 healthy control volunteers, age and gender matched, were also collected from a similar population. We specifically chose samples from the primary tumor of each original specimen where cancer cells were identified by the pathologists. Demographic, preoperative and postoperative data were available for all patients. The patients were staged according to the International Union Against Cancer (16). The study protocol was approved by both the Ethics Committees of Evangelismos General Hospital and the Ethics Board of Medical School, University of Athens. All participants provided informed consent prior to entry to the study.

Extraction of genomic DNA. All samples were formalinfixed and paraffin-embedded, and genomic DNA was extracted using the QIAmp extraction DNA kit (Qiagen $\mathrm{GmbH}$, Hilden, Germany) according to the manufacturer's protocols. Concentration of the genomic DNA was assessed by the GenQuant spectrometer (Pharmacia Biotech Inc., Piscataway, NJ).

PCR analysis and DNA sequencing. Nested PCR was used for the detection of any point mutations in exons 2 and 3 of the
HRAS gene, followed by direct sequencing. The sequences of the 2 oligonucleotide primer pairs were as follows: (1a) exon 2 forward 5'-ATCACTGGGTCATTAAGACAAGTGG-3' and exon 2 reverse 3'-CAGGCTCACCTCTATAGTGGGGTC-5', and (1b) exon 2 nested forward 5'-AGGTAGGCACGCTG CAGTCCTT-3' and exon 2 nested reverse 3'-AAATGGTTC TGGATCAGCTGGATG-5' (2a) exon 3 forward 5'-ATGAGA GGTACCAGGGAGAGGCT-3' and exon 3 reverse 3'-TGT ACTGGTGGATGTCCTCAAAAGACT-5' (2b) exon 3 nested forward 5'-ATTCCTACCGGAAGCAGGTGGTCAT-3' and exon 3 nested reverse 3'-AGACTTGGTGTTGTTGATGGCA AAC-5' (3a) exon 15 BRAF forward 5'-TCTTCATAATGCT TGCTCTGATAGG-3' exon 15 reverse 3'-CAGCATCTCAGG GCCAAAAATT-5' and (3b) exon 15 nested forward 5'-TAG GAAAATGAGATCTACTGTTTTCCTT-3' and exon 15 nested reverse 3'-TACCATCCACAAAATGGATCCAGAC-5'. The nested polymerase chain reaction (PCR) reaction was carried out in a total volume of $25 \mu \mathrm{l}$ in a PTC-200 Peltier Thermal Cycler (MJ Research, Inc., Waltham, MA). The mixture of the PCR reaction contained $2.5 \mu \mathrm{l} 10 \mathrm{X}$ reaction buffer, $3 \mu \mathrm{l}$ dNTP (2.5 mM of each dNTP), $1.75 \mu 1$ forward primer $(10 \mu \mathrm{M}), 1 \mu \mathrm{l}$ reverse primer (25 pmol), $0.75 \mu \mathrm{l} \mathrm{MgCl}_{2}$ (50 mM), $0.25 \mu \mathrm{l}$ Platinum Taq DNA polymerase ( $5 \mathrm{U} / \mu \mathrm{l})$, and $2 \mu \mathrm{l}$ DNA template. For the nested PCR, DNA template was diluted 1:50 and one of the final concentration was used. Amplification was carried out with $5 \mathrm{~min}$ of initial denaturation at $94^{\circ} \mathrm{C}$ followed by 36 cycles of denaturation at $94^{\circ} \mathrm{C}$ for $45 \mathrm{sec}$, primer annealing at $55^{\circ} \mathrm{C}$ for the BRAF and at $58^{\circ} \mathrm{C}$ for the HRAS for $45 \mathrm{sec}$, and extension at $72^{\circ} \mathrm{C}$ for $45 \mathrm{sec}$ and final extension at $72^{\circ} \mathrm{C}$ for $3 \mathrm{~min}$. The PCR products were subsequently analyzed on a 4200 Two-Dye DNA Analysis System (LI-COR Biosciences, Lincoln, NE). (HRAS accession no. BC095471 and BRAF accession no. BC1120791).

Statistical analysis. All the available data were entered into a database using SPSS statistical software (SPSS 15.0 for Windows; SPSS Inc., Chicago, IL). The $\chi^{2}$ test was also used for a comparison of qualitative variables. The P-value was found to be significant when it was $<0.05$.

\section{Results}

Out of the 86 patients, $17(19.8 \%)$ were stage I, 12 (14\%) were stage II, $14(16.3 \%)$ were stage III and $43(50 \%)$ were stage IV, according to pTNM stage. Thirty-one patients (36\%) had cancer of the oral tongue, $8(9.3 \%)$ of the floor of the mouth, $25(29.1 \%)$ of the lower gingival and alveolus, $4(4.7 \%)$ of the upper gingival, 9 (10.5\%) of the buccal mucosa, $6(7 \%)$ of the retromolar triangle and $3(3.5 \%)$ patients had cancer of the lip mucosa. The histological grade was well-differentiated in 17 (21\%) of the patients, moderately-differentiated in $56(69.1 \%)$ of the patients and poorly-differentiated in $8(9.9 \%)$ of the patients. The clinical characteristics of the patients studied and the genetic alterations of the BRAF and HRAS gene are summarized in Table I.

The V600E hotspot mutation was not detected in any of the 86 specimens analyzed. Nevertheless, a new silent mutation/ polymorphism was detected in 10 out of 86 specimens $(8.6 \%)$ (accession no. BC1120791). This consists a novel finding and was entered in the Ensemble database (ssbraf-172036). It refers 


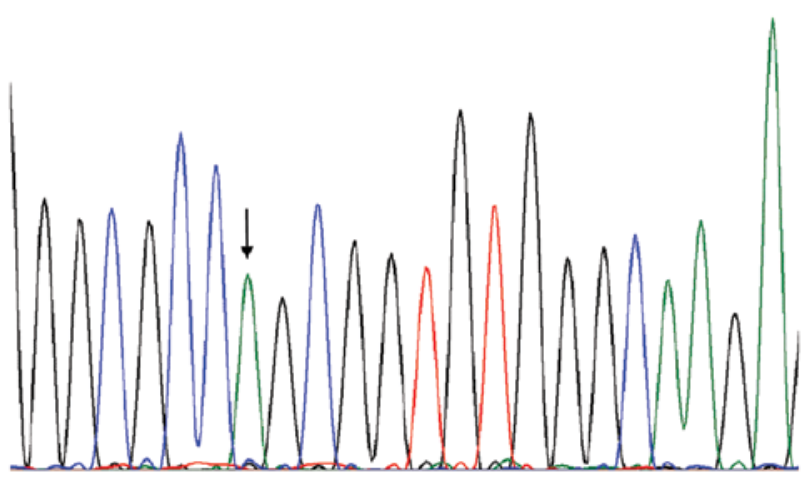

Figure 1. Gly12Ser. Hotspot codon 12 exon 2. Sequence chromatogram fragment of the HRAS gene exon 2 as determined by automated sequence analysis. The arrow indicates the substitution of a guanine by an adenine in a homozygous sample (accession no. BC095471.1).

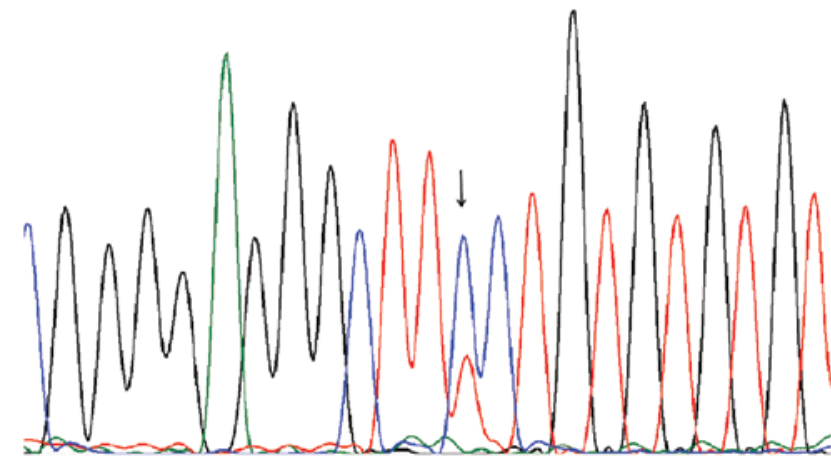

Figure 2. Phenylalanine78Phenylalanine(F78F) exon 3. Sequence chromatogram fragment of the HRAS gene as determined by automated sequence analysis. The arrow indicates the 2 peaks detected (both wild-type cytosine and substitution by a thymine) in a heterozygous sample (accession no. BC095471.1).

to exon 15 , where thymine is substituted by a cytosine (cDNA position T1803C) and results in a silent mutation. All specimens harbouring this polymorphism were heterozygous. This polymorphism was found in none of the 80 controls specimens $(\mathrm{P}<0.001)$.

Out of 86 tumors $2(2.3 \%)$ had a hotspot mutation in codon 12 of the HRAS gene (Gly12Ser) (accession no. BC095471.1) (Fig. 1). Four new mutations were detected. Two of the new mutations harboured HRAS exon 2. Both were $\mathrm{C} \rightarrow \mathrm{T}$ transitions. One was a silent mutation (phenylalanine to phenylalanine, Phe78Phe) (Fig. 2) harbouring exon 3, while the other was a missense mutation (Alanine to Valine) Ala18Val harbouring exon 2 (accession no. BC095471.1) (Fig. 3). The specimen harbouring this missense mutation was homozygous. Another mutation harboured the 5'-UTR (Cytosine149Thymine) of the same exon (accession no. BC095471.1) (Fig. 4). It was a $\mathrm{C} / \mathrm{T}$ (Cytosine/Thymine) transition and the specimen was

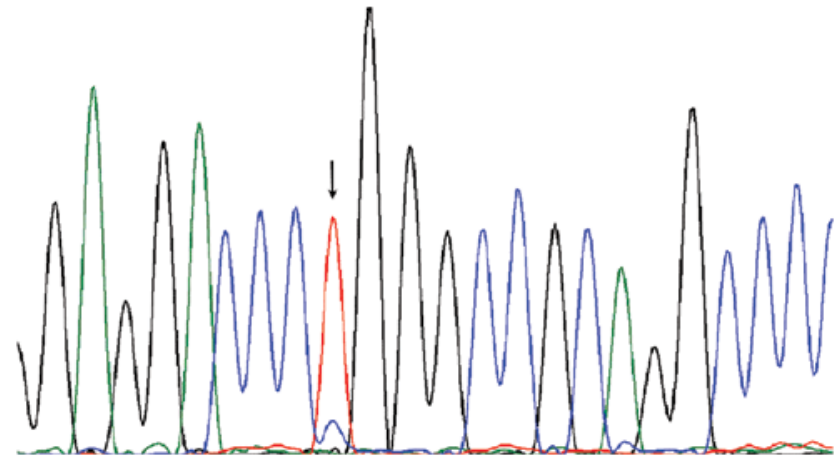

Figure 3. Ala18Val exon 2. Sequence chromatogram fragment of the HRAS gene as determined by automated sequence analysis. The arrow indicates the substitution of cytosine by a thymine in a homozygous sample (accession no. BC095471.1).

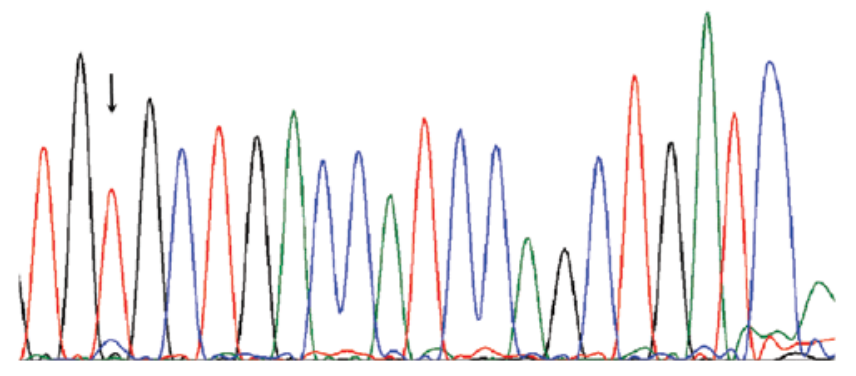

Figure 4. C149T exon 2. Sequence chromatogram fragment of the HRAS gene as determined by automated sequence analysis. The arrow indicates the substitution of cytosine by a thymine in a homozygous sample (accession no. BC095471.1).

homozygous for it. Another mutation was located in the intravening sequence 1 (G3542A). The specimen harbouring this mutation was heterozygous. It should be noted that one patient harboured both the new missense mutation Ala18Val and the 5'-UTR mutation homozygously. To our knowledge, there is no information available in the literature on these mutations. As these mutations are not stored in the COSMIC (Catalog Of Somatic Mutations In Cancer), a database of Sanger Institute, $\mathrm{UK}$, this is the first report of such mutations in OSCC.

Furthermore, three already registered single nucleotide polymorphisms harbouring HRAS exon 2 were detected. The polymorphism rs61877782 was found in $2.3 \%$ of the specimens ( 2 of 86). The polymorphism rs 41294870 was found in 3 out of 86 tumors $(3 \%)$, whereas the polymorphism rs112587690 was found in 12 out of 86 tumors (14\%).

\section{Discussion}

The mutation frequency of BRAF has been reported to be $59 \%$ in melanomas, $18 \%$ in colorectal cancers and $14 \%$ in 
Table I. Clinical characteristics and HRAS and BRAF genetic alterations in patients with oral squamous cell carcinoma.

\begin{tabular}{|c|c|c|c|c|c|c|c|}
\hline $\begin{array}{l}\text { Case } \\
\text { no. }\end{array}$ & Gender & Age & Tumor location & Histological grade & Stage & $\begin{array}{c}\text { HRAS } \\
\text { (accession no. BC095471) }\end{array}$ & $\begin{array}{c}\text { BRAF } \\
\text { (accession no. BC1120791) }\end{array}$ \\
\hline 1 & M & 56 & Floor of mouth & Moderate & IV & & \\
\hline 2 & $\mathrm{~F}$ & 54 & Floor of mouth & Moderate & IV & & \\
\hline 3 & $\mathrm{~F}$ & 54 & Buccal mucosa & Moderate & IV & & \\
\hline 4 & $\mathrm{~F}$ & 71 & Upper gingiva & - & II & & ssBRAF-172036 \\
\hline 5 & $\mathrm{~F}$ & 69 & Lower gingiva & Poor & IV & & \\
\hline 6 & M & 66 & Floor of mouth & Moderate & IV & & \\
\hline 7 & $\mathrm{~F}$ & 56 & Lower gingiva & Moderate & IV & & \\
\hline 8 & M & 43 & Retromolar triangle & Well & I & rs112587690 & \\
\hline 9 & $\mathrm{~F}$ & 70 & Buccal mucosa & Moderate & III & & \\
\hline 10 & $\mathrm{~F}$ & 78 & Oral tongue & Moderate & II & & \\
\hline 11 & M & 80 & Lower gingiva & Moderate & IV & & \\
\hline 12 & $\mathrm{~F}$ & 26 & Oral tongue & Well & III & & \\
\hline 13 & $\mathrm{~F}$ & 48 & Oral tongue & Moderate & III & & ssBRAF-172036 \\
\hline 14 & M & 41 & Buccal mucosa & Moderate & III & & ssBRAF-172036 \\
\hline 15 & $\mathrm{~F}$ & 91 & Lip mucosa & Moderate & III & & ssBRAF-172036 \\
\hline 16 & $\mathrm{~F}$ & 65 & Lip mucosa & Moderate & I & & ssBRAF-172036 \\
\hline 17 & $\mathrm{~F}$ & 72 & Lower gingiva & Moderate & I & rs112587690 & \\
\hline 18 & M & 45 & Floor of mouth & Well & I & & \\
\hline 19 & M & 45 & Lower gingiva & - & IV & & \\
\hline 20 & $\mathrm{~F}$ & 79 & Oral tongue & Poor & IV & & \\
\hline 21 & M & 67 & Upper gingiva & Moderate & IV & & \\
\hline 22 & $\mathrm{~F}$ & 30 & Oral tongue & Well & IV & & \\
\hline 23 & M & 65 & Retromolar triangle & Moderate & III & & \\
\hline 24 & M & 65 & Retromolar triangle & Moderate & III & & ssBRAF-172036 \\
\hline 25 & $\mathrm{~F}$ & 75 & Oral tongue & Moderate & IV & & \\
\hline 26 & $\mathrm{~F}$ & - & Lower gingiva & Moderate & IV & & \\
\hline 27 & M & 74 & Retromolar triangle & Moderate & IV & & \\
\hline 28 & $\mathrm{~F}$ & 54 & Buccal mucosa & - & IV & rs 112587690 & \\
\hline 29 & M & 69 & Lower gingiva & Moderate & IV & & \\
\hline 30 & $\mathrm{~F}$ & 76 & Retromolar triangle & Moderate & IV & & \\
\hline 31 & M & 55 & Lip mucosa & Moderate & I & & ssBRAF-172036 \\
\hline 32 & M & 51 & Lower gingiva & Well & IV & & \\
\hline 33 & $\mathrm{~F}$ & 61 & Oral tongue & Moderate & IV & & \\
\hline 34 & $\mathrm{~F}$ & 69 & Oral tongue & Poor & III & & ssBRAF-172036 \\
\hline 35 & $\mathrm{~F}$ & 87 & Buccal mucosa & Well & I & & \\
\hline 36 & M & 64 & Upper gingiva & Well & I & rs112587690 & \\
\hline 37 & M & 24 & Oral tongue & Moderate & IV & & \\
\hline 38 & M & 62 & Oral tongue & Moderate & III & & \\
\hline 39 & M & 57 & Oral tongue & Moderate & III & & \\
\hline 40 & M & 73 & Floor of mouth & Moderate & IV & & \\
\hline 41 & $\mathrm{~F}$ & 74 & Lower gingiva & Moderate & IV & & \\
\hline 42 & M & 31 & Oral tongue & Moderate & $\mathrm{I}$ & & \\
\hline 43 & M & 55 & Floor of mouth & Moderate & III & & \\
\hline 44 & $\mathrm{~F}$ & - & Buccal mucosa & Moderate & I & & \\
\hline 45 & M & 58 & Floor of mouth & Moderate & IV & & \\
\hline 46 & $\mathrm{~F}$ & 63 & Buccal mucosa & Moderate & II & rs 112587690 & \\
\hline 47 & $\mathrm{~F}$ & 73 & Retromolar triangle & Moderate & II & & \\
\hline 48 & M & 44 & Oral tongue & Well & IV & & \\
\hline 49 & $\mathrm{~F}$ & 78 & Oral tongue & Moderate & IV & & \\
\hline 50 & M & 61 & Lower gingiva & Moderate & IV & & \\
\hline 51 & M & 61 & Lower gingiva & Moderate & IV & & ssBRAF-172036 \\
\hline
\end{tabular}


Table I. Continued.

\begin{tabular}{|c|c|c|c|c|c|c|c|}
\hline $\begin{array}{l}\text { Case } \\
\text { no. }\end{array}$ & Gender & Age & Tumor location & Histological grade & Stage & $\begin{array}{c}\text { HRAS } \\
\text { (accession no. BC095471) }\end{array}$ & $\begin{array}{c}\text { BRAF } \\
\text { (accession no. BC1120791) }\end{array}$ \\
\hline 52 & M & 73 & Lower gingiva & Moderate & IV & & \\
\hline 53 & M & 37 & Lower gingiva & Moderate & IV & & \\
\hline 54 & $\mathrm{~F}$ & 79 & Lower gingiva & Well & IV & & \\
\hline 55 & M & 46 & Oral tongue & Poor & IV & & \\
\hline 56 & $\mathrm{~F}$ & 72 & Oral tongue & Poor & II & & \\
\hline 57 & $\mathrm{~F}$ & 72 & Lower gingiva & Moderate & I & G3542A & \\
\hline 58 & $\mathrm{~F}$ & 48 & Lower gingiva & Poor & IV & rs61877782 & \\
\hline 59 & $\mathrm{~F}$ & 26 & Oral tongue & Well & III & & \\
\hline 60 & $\mathrm{~F}$ & 47 & Oral tongue & Moderate & II & & \\
\hline 61 & M & 46 & Oral tongue & Moderate & II & & ssBRAF-172036 \\
\hline 62 & M & 57 & Oral tongue & Poor & IV & & \\
\hline 63 & $\mathrm{~F}$ & 76 & Lower gingiva & Moderate & III & F78F & \\
\hline 64 & $\mathrm{~F}$ & 81 & Oral tongue & Moderate & I & Hotspot codon 12 & \\
\hline 65 & M & 65 & Lower gingiva & Well & I & & \\
\hline 66 & $\mathrm{~F}$ & 73 & Oral tongue & Moderate & II & rs61877782 & \\
\hline 67 & $\mathrm{~F}$ & 56 & Lower gingiva & Moderate & IV & & \\
\hline 68 & $\mathrm{~F}$ & 68 & Oral tongue & Well & $\mathrm{I}$ & & \\
\hline 69 & $\mathrm{~F}$ & 74 & Buccal mucosa & Moderate & IV & & \\
\hline 70 & M & 75 & Oral tongue & Moderate & IV & rs 112587690 & \\
\hline 71 & $\mathrm{~F}$ & 68 & Upper gingiva & Moderate & IV & rs 112587690 & \\
\hline 72 & $\mathrm{~F}$ & 66 & Oral tongue & Moderate & II & Hotspot codon 12 & \\
\hline 73 & M & 62 & Lower gingiva & Moderate & $\mathrm{I}$ & & \\
\hline 74 & M & 62 & Floor of mouth & Well & $\mathrm{I}$ & rs41294870 & \\
\hline 75 & $\mathrm{~F}$ & 60 & Lower gingiva & Poor & IV & & \\
\hline 76 & $\mathrm{~F}$ & 82 & Lower gingiva & Moderate & IV & & \\
\hline 77 & $\mathrm{~F}$ & 75 & Oral tongue & Well & $\mathrm{I}$ & rs41294870 & \\
\hline 78 & M & 70 & Buccal mucosa & Moderate & IV & & \\
\hline 79 & M & 58 & Oral tongue & Moderate & III & A18V,C149T & \\
\hline 80 & M & 50 & Oral tongue & Well & $\mathrm{I}$ & & \\
\hline 81 & M & 52 & Oral tongue & Well & II & rs112587690 & \\
\hline 82 & M & 57 & Lower gingiva & Moderate & II & rs41294870 & \\
\hline 83 & M & 64 & Lower gingiva & Moderate & II & rs 112587690 & \\
\hline 84 & M & 51 & Lower gingiva & Well & IV & rs 112587690 & \\
\hline 85 & M & 55 & Oral tongue & - & IV & rs 112587690 & \\
\hline 86 & M & 69 & Oral tongue & - & IV & rs 112587690 & \\
\hline
\end{tabular}

liver cancers (13). Weber et al analysed the BRAF mutation status in HNSCC of various sites in Germany by the use of PCR and sequencing and reported a 3\% mutation frequency in their pharynx and hypopharynx specimens but none in specimens of the oral cavity (18). Davies et al analyzed 19 primary HNSCC using capillary-based modified heteroduplex mutation detection method in exons 11 and 15 and found no mutations (13). Al Sheikh Ali et al reported lack of BRAF mutations in 91 Japanese HNSCC as well as 12 HNSCC cell lines by SSCP and MASA analyses (19). Shelly et al conducted a study in canine oral cancer specimens and found no BRAF mutation in their cohort of samples (20). There is limited data regarding the implication of BRAF in OSCCs. Bruckman et al performed a study in the USA in OSCC tumors and reported a frequency of $2 \%(21)$.
RAS genes have been found to be activated in a wide variety of human tumors, including colon carcinoma and pancreatic carcinoma for which a 40 and $95 \%$ incidence of RAS gene activation has been demonstrated, respectively $(22,23)$. However, the incidence of RAS gene mutation is low in breast cancer and stomach cancer $(24,25)$. A high incidence of RAS mutation, 18-30\% of cases from India and Vietnam, has been reported and it has been associated with areca nut chewing $(3,10,26)$ whereas lower frequencies of $4-5 \%$ were reported from UK and Japan (27-29).

The present study showed lack of BRAF mutations in OSCC in the Greek population. This is in accordance with previous studies showing minor or no contribution of the BRAF mutations to the HNSCC $(12,17,18)$ in the German and Japanese population and a minor contribution of the BRAF 
gene to the OSCC in a study conducted in the USA (20). The new polymorphism (T1803C) was detected at a frequency of $8.6 \%$ in the cancer specimens and in no specimen in the control group. No correlation was found between the polymorphisms and age, gender, stage, histological grade and prognosis. Thus, further studies with larger sample sizes are necessary in order to elucidate statistical differences and delineate any potential role of this silent mutation/polymorphism.

Hotspot mutations in the HRAS gene were found in two cases $(2.3 \%)$, which consists a relatively low frequency, all of them harbouring codon 12 . Nevertheless, another four mutations were detected in our specimen, resulting in an overall frequency of HRAS genetic alterations of $4.7 \%$. None of them have been previously reported. Although, only one of them resulted in an amino acid change (Ala18Val), further studies are needed in order to test whether the other mutations detected in the exon 2 (Phe78Phe), in the 5'-UTR region (untranslated region) or in the intronic region have any impact on the RNA splicing or the regulation of the gene and to further examine the function of the protein encoded. Nevertheless, these genetic alterations probably demonstrate the stress imposed on the gene by carcinogens.

Since no BRAF mutations were detected, no specimen harboured the BRAF and HRAS hotspot mutations. Furthermore, the same specimens have been previously examined for PIK3CA hotspot mutations, but none have been detected (15). Therefore, it is possible that the upstream effector HRAS may contribute to the activated PI3K signalling pathway as previously reported $(7,8)$.

\section{Acknowledgements}

The authors would like to thank the Pathology Department of Evangelismos Hospital for accurately selecting the sections with cancerous tissue of the specimens studied. The authors would also like to thank the State Scholarships Foundation of Greece.

\section{References}

1. Kademani D: Oral cancer. Mayo Clin Proc 82: 878-887, 2007.

2. Franceschi S, Talamini R, Barra S, et al: Smoking and drinking in relation to cancers of the oral cavity, pharynx, larynx, and esophagus in northern Italy. Cancer Res 50: 6502-6507, 1990.

3. Murugan AK, Hong NT, Cuc TT, et al: Detection of two novel mutations and relatively high incidence of H-RAS mutations in Vietnamese oral cancer. Oral Oncol 45: e161-e166, 2009.

4. Miller CS and White DK: Human papillomavirus expression in oral mucosa, premalignant conditions, and squamous cell carcinoma: a retrospective review of the literature. Oral Surg Oral Med Oral Pathol Oral Radiol Endod 82: 57-68, 1996.

5. Lyronis ID, Baritaki S, Bizakis I, et al: K-ras mutation, HPV infection and smoking or alcohol abuse positively correlate with esophageal squamous carcinoma. Pathol Oncol Res 14: 267-273, 2008.

6. Kiaris H and Spandidos DA: Mutations of ras genes in human tumours. Int J Oncol 7: 413-429, 1995.

7. Barbacid M: RAS genes. Annu Rev Biochem 56: 779-827, 1987.

8. Kozaki K, Imoto I, Pimkhaokham A, et al: PIK3CA mutation is an oncogenic abberation at advanced stages of oral squamous cell carcinoma. Cancer Sci 97: 1351-1358, 2006.
9. Pedrero JM, Carracedo DG, Pinto CM, et al: Frequent genetic and biochemical alterations of the PI 3-K/AKT/PTEN pathway in head and neck squamous cell carcinoma. Int J Cancer 114: 242-248, 2005.

10. Paterson IC, Eveson JW and Prime SS: Molecular changes in oral cancer may reflect aetiology and ethnic origin. Eur J Cancer B Oral Oncol 32: 150-153, 1996.

11. Saranath D, Chang SE, Bhoite LT, et al: High frequency mutation in codons 12 and 61 of H-ras oncogene in chewing tobacco-related human oral carcinoma in India. Br J Cancer 63: 573-578, 1991.

12. Kuo MY, Jeng JH, Chiang CP, et al: Mutations of Ki-ras oncogene codon 12 in betel quid chewing-related human oral squamous cell carcinoma in Taiwan. J Oral Pathol Med 23: 70-74, 1994.

13. Davies H, Bignell GR, Cox C, et al: Mutations of the BRAF gene in human cancer. Nature 417: 949-954, 2002.

14. Wan PT, Garnett MJ, Roe SM, et al: Mechanism of activation of the RAF-ERK signaling pathway by oncogenic mutations of B-RAF. Cell 116: 855-867, 2004.

15. Jaworski M, Buchmann A, Bauer P, et al: B-raf and Ha-ras mutations in chemically induced mouse liver tumors. Oncogene 24: 1290-1295, 2005.

16. Kostakis GC, Papadogeorgakis N, Koumaki V, et al: Absence of hotspot mutations in exons 9 and 20 of the PIK3CA gene in human oral squamous cell carcinoma in the Greek population. Oral Surg Oral Med Oral Pathol Oral Radiol Endod 109: e53-e58, 2010.

17. Booth PW, Schendel SA and Hausamen JE: Maxillofacial Surgery. 2nd edition. Churchill Livingstone, London, pp299-304, 2007.

18. Weber A, Langhanki L, Sommerer F, et al: Mutations of the BRAF gene in squamous cell carcinoma of the head and neck. Oncogene 22: 4757-4759, 2003.

19. Al Sheikh Ali M, Gunduz M, Gunduz E, et al: Lack of B-RAF mutations in head and neck squamous cell carcinoma. Folia Biol (Praha) 54: 157-161, 2008.

20. Shelly S, Chien MB, Yip B, et al: Exon 15 BRAF mutations are uncommon in canine oral malignant melanomas. Mamm Genome 16: 211-217, 2005.

21. Bruckman KC, Schönleben F, Qiu W, et al: Mutational analyses of the BRAF, KRAS, and PIK3CA genes in oral squamous cell carcinoma. Oral Surg Oral Med Oral Pathol Oral Radiol Endod 110: 632-637, 2010.

22. Bos JL, Fearon ER, Hamilton SR, et al: Prevalence of ras gene mutations in human colorectal cancers. Nature 327: 293-297, 1987.

23. Almoguera C, Shibata D, Forrester K, et al: Most human carcinomas of the exocrine pancreas contain mutant c-K-ras genes. Cell 53: 549-554, 1988.

24. Kraus MH, Yuasa Y and Aaronson SA: A position 12-activated H-ras oncogene in all HS578T mammary carcinosarcoma cells but not normal mammary cells of the same patient. Proc Natl Acad Sci USA 81: 5384-5388, 1984.

25. Theillet C, Lidereau R, Escot C, et al: Loss of a c-H-ras-1 allele and aggressive human primary breast carcinomas. Cancer Res 46: 4776-4781, 1986.

26. Munirajan AK, Mohanprasad BK, Shanmugam G and Tsuchida N: Detection of a rare point mutation of codon 59 and relatively high incidence of $\mathrm{H}$-ras mutation in Indian oral cancer. Int J Oncol 13: 971-974, 1998.

27. Chang SE, Bhatia P, Johnson NW, et al: Ras mutations in United Kingdom examples of oral malignancies are infrequent. Int $\mathrm{J}$ Cancer 48: 409-412, 1991.

28. Sakai E, Rikimaru K and Ueda M: The p53 tumor-suppressor gene and ras oncogene mutations in oral squamous-cell carcinoma. Int J Cancer 52: 867-872, 1992.

29. Kiaris H, Spandidos DA, Jones AS, Vaughan ED and Field JK: Mutations, expression and genomic instability of the H-ras protooncogene in squamous cell carcinomas of the head and neck. Br J Cancer 72: 123-128, 1995. 